\title{
АРИФМЕТИЧЕСКИЕ ИНВАРИАНТЫ ОДНОГО КЛАССА АЛГЕБРАИЧЕСКИХ ТОРОВ
}

Пусть задано произвольное поле ł. Через $\bar{f}$ будем обозначать его алгебраическое замыкание, а символом $\overline{\mathfrak{f}^{*}}$ - мультипликативную группу поля f. Пусть $F=\operatorname{Gal}(\overline{\mathrm{f}} / \mathfrak{f})$ - группа Галуа $\overline{\mathfrak{f}}$ над f́. Напомним, что алгебраическая группа $T$, определенная над полем $\mathfrak{f}$, называется тором, если тензорное произведение $T(\overline{\mathrm{f}})=T \otimes_{\mathfrak{f}} \overline{\mathfrak{f}}$ изоморфно расслоенному произведению $\overline{\mathfrak{f}}^{*} \times \ldots \times \overline{\mathfrak{f}}^{*}$. Число членов в произведении называется размерностью тора. Группа рациональных характеров $\hat{T}=\operatorname{Hom}\left(T, \overline{1^{*}}\right)$ является Z-свободным $F$-модулем и $\hat{\hat{T}}=T$.

В работах Т. Оно $\left[{ }^{1}\right]$ и В. Е. Воскресенского $\left[{ }^{2,3}\right]$ был достигнут ряд глубоких результатов в теории алгебраических торов над числовым

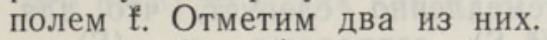

I. T. Оно [ $\left.{ }^{1}\right]$ нашел формулу для числа Тамагавы $\tau(T)$ тора $T$. Оказывается,

$$
\tau(T) \cdot[\amalg(T)]=\left[H^{1}(\mathfrak{f}, \hat{T})\right] .
$$

Здесь Ш $(T)$ - группа Шафаревича-Тэйта тора $T$, и определяется она как ядро естественного отображения $H^{1}(\mathfrak{f}, T) \rightarrow \prod_{v}^{1} H^{1}\left(\mathfrak{f}_{v}, T\right)$, где произведение берется по всем нормированиям $v$ поля ‡. Через $H^{1}(\mathfrak{f}, T)$ и $H^{1}\left(\mathfrak{f}_{v}, T\right)$ обозначены группы $\left.H^{1}(F, T \overline{(\mathfrak{f}})\right)$ и $H^{1}\left(\mathrm{Gal}\left(\overline{\mathfrak{f}_{w}} / \mathfrak{f}_{v}\right), T\left(\overline{\mathfrak{f}_{w}}\right)\right)$ соответственно, где $w-$ продолжение нормирования $v$ с $\mathfrak{f}$ на $\bar{f}$. Квадратные скобки в формуле означают порядок. Определение числа $\tau(T)$ нам не понадобится, его можно найти в $\left[{ }^{1,4}\right]$.

II. В. Е. Воскресенский $\left[{ }^{2}\right]$ получил для тора $T$ следующую точную последовательность, связывающую его арифметические инварианты:

$$
0 \rightarrow A(T) \rightarrow H^{1}(\mathfrak{f}, \mathrm{Pic} \bar{V}(T))^{\circ} \rightarrow \amalg(T) \rightarrow 0 .
$$

Здесь $A(T)$ - ядро слабой аппроксимации в группе $T$, которое определяется как фактор-пространство $\left(\boldsymbol{\Pi}_{v} T\left(\mathfrak{f}_{v}\right)\right) / \overline{T(\mathfrak{f})} \cdot(\overline{T(\mathfrak{f})})-$ замыкание группы $T(\mathfrak{f})$ при ее диагональном вложении в прямое произведение $\prod_{v} T\left(\mathfrak{f}_{v}\right)$, где $v$ пробегает все нормирования $\mathfrak{f} \operatorname{Pic} \bar{V}(T)$ группа Пикара замыкания неособой минимальной проективной модели тора $T$. Определение ее дано в $\left[{ }^{2}\right]$. Символом «о» обозначена двойственность в смысле Понтрягина. 
В вышеуказанных работах арифметические инварианты $\tau(T)$, $H^{1}(\mathfrak{f}, \operatorname{Pic} \bar{V}(T)), A(T)$ вычислены в некоторых специальных случаях. Все эти результаты мы применим к одному классу алгебраических торов, а именно к таким торам, которые возникают в задаче погружения числовых полей с абелевым ядром. Это позволит передоказать ряд полученных ранее в этой задаче результатов и получить некоторые новые факты.

Пусть $A$ - конечная абелева группа с $F$-модульной структурой, $\mathrm{Z}[\hat{A}]-$ групповое кольцо группы $\hat{A}=\operatorname{Hom}\left(A, \overline{\mathfrak{f}^{*}}\right)$, являющейся в этом случае также $F$-модулем. Z[A $]$ тогда $Z$-свободный $F$-модуль с образующими $\left\{l_{a}\right\}$, которые находятся во взаимно-однозначном соответствии с элементами $a \in \hat{A}$. Действие $F$ на нем задается формулой $\left(l_{a}\right)^{f}=l_{a}, f \in F$. Рассмотрим теперь точную последовательность модулей

$$
0 \rightarrow \hat{S} \rightarrow Z[\hat{A}] \stackrel{\mu}{\rightarrow} \hat{A} \rightarrow 0,
$$

где гомоморфизм $\mu$ переводит образующую $l_{a}$ в $a$, а $\hat{S}=\operatorname{ker} \mu$. Обозначим через $R$ выражение $\mathrm{Z}[\hat{A}]$. Последовательность (1) будет определять тогда двойственную последовательность торов над $⿱ 亠 䒑$ :

$$
1 \rightarrow A \rightarrow R \rightarrow S \rightarrow 1 \text {. }
$$

Как показано в $\left[{ }^{5}\right]$, последовательность (2) возникает в задаче погружения полей с абелевым ядром. Для нас особый интерес представляет группа $H^{1}(\mathfrak{f}, S)$. Если $H^{1}(\mathfrak{f}, S)=0$, то необходимое условие разрешимости задачи погружения - условие согласности Фаддеева-Хассе является и достаточным. Легко показать, что $H^{1}(\mathfrak{f}, \hat{S})=0$ и $H^{1}\left(\mathfrak{f}_{v}, S\right)=H^{1}\left(\mathfrak{f}_{v}, \hat{S}\right)=0$. Из этого немедленно следует, что для нашего тора $S$ группы Ш $(S)$ и $H^{1}(\mathfrak{f}, S)$ совпадают, а $\tau(S)=$ $=1 /\left[H^{1}(\mathfrak{f}, S)\right]$. Сформулируем эти факты в виде теоремы.

Т е о рем а 1. А. $H^{1}(\mathfrak{f}, S)=\amalg(S)$;

$$
\text { Б. } \tau(S) \cdot\left[H^{1}(\mathfrak{f}, S)\right]=1 \text {. }
$$

Заметим, что точная последовательность из II для тора $S$ принимает вид

$$
0 \rightarrow A(S) \stackrel{\vartheta}{\rightarrow} H^{1}(\mathfrak{f}, \mathrm{Pic} \bar{V}(S))^{\circ} \rightarrow H^{1}(\mathrm{f}, S) \rightarrow 0 .
$$

Следствие. Условие согласности достаточно для разрешимости задачи погружения, если:

1) тор $S$ рационален над $\mathfrak{f}$;

2) $S$ имеет циклическое поле расщепления;

3) у тора $S$ существует поле расщепления $\mathfrak{\&}$ такое, что в $\mathrm{Gal}(\mathfrak{Q} / \mathfrak{f})$ все силовские подгруппы циклические;

4) модуль рациональных характеров $\hat{S}-$ проективен;

5) мономорфизм $\gamma$ является изоморфизмом.

Пп. 1 и 2 следуют из того, что, как показано в $\left[{ }^{2}\right]$, в этих случаях $H^{1}(\mathfrak{f}, \mathrm{Pic} \bar{V}(S))=0$, и из последовательности (3). В работе же $\left[{ }^{3}\right]$ аналогичный результат получен для тора из п. 3. П. 4 получается как следствие результата Т. Оно [ $\left.{ }^{1}\right]$. В этом случае $\tau(S)=1$.

3 а меч ан ие 1. T. Оно [ $\left.{ }^{1}\right]$ строит тор с нецелым числом Тамагавы и с помощью этого построения находит пример полупростой алгебраической группы, число Тамагавы которой нецелое, и таким обра- 
зом дает отрицательный ответ на вопрос, поставленный А. Вейлем в $\left[{ }^{4}\right]$. Давно существуют примеры задач погружения с абелевым ядром $[6,7]$, где согласность для разрешимости не достаточна. Очевидно, в этих случаях для тора $S$ группа $H^{1}(\mathfrak{f}, S) \neq 0$, а из теоремы 1 следует тогда, что $\tau(S)=1 / n$, где $n-$ порядок $H^{1}(\mathfrak{f}, S)$. Таким образом, здесь также можно указать торы с нецелым числом Тамагавы.

Из вышесказанного видно, что почти все результаты из теоремы 1 получаются потому, что в некоторых случаях удается вычислить группу $H^{1}(\mathfrak{f}$, Ріс $\bar{V}(S))$. Если $A(S)=0$, то $H^{1}(\mathfrak{f}, \mathrm{Pic} \bar{V}(S))^{\circ}=H^{1}(\mathfrak{f}, S)$. Спрашивается: как часто $A(S)=0$ ? Мы приведем здесь один критерий, который, вероятно, может быть полезен при выяснении этого вопроса.

Т е о р е а 2. Пусть $s_{0}$ - конечное множество нормирований поля группы разложения которых нециклические. Тогда $A(S)=0$ тогда и только тогда, когда естественное отображение $\alpha_{1}: H^{1}(\mathfrak{f}, A) \rightarrow \prod_{v \in s_{0}} H^{1}\left(\mathfrak{f}_{v}, A\right)$ является эпиморфизмом.

Доказ ательство. Не обходи мость: пусть $A(S)=0$. Рассмотрим следующую коммутативную диаграмму, которая индуцируется последовательностью (2):

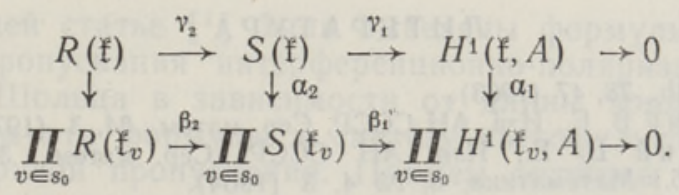

Крайние правые члены в этой диаграмме равны нулю, так как легко показать, что $H^{1}(\mathfrak{f}, R)=H^{1}\left(\mathfrak{f}_{v}, R\right)=0$. Гомоморфизмы $\beta_{1}$ и $\gamma_{1}$ являются эпиморфизмами, и $\beta_{2}$ открыт в $v$-адической топологии. Следовательно, существует такая окрестность единицы $\mathfrak{B}$ в группе $\prod_{v \in s_{0}} S\left(\mathfrak{f}_{v}\right)$, что $\beta_{1}(\mathfrak{B})=1$. Так как $A(S)=0$ по условию, то $\prod_{v \in s_{0}} S\left(\mathfrak{f}_{v}\right)=\mathfrak{B} \alpha_{2}(S(\mathfrak{f}))$. Значит, $\beta_{1} \alpha_{2}=\alpha_{1} \gamma_{1}-$ эпиморфизм, но $\gamma_{1}-$ эпиморфизм, следовательно, $\alpha_{1}$ - эпиморфизм.

Д ост аточность: пусть $\alpha_{1}$ - эпиморфизм. По результату В. Е. Воскресенского $\left[{ }^{2}\right] A(T)=A\left(T, s_{0}\right)$ для произвольного тора $T$, где $A\left(T, s_{0}\right)=\prod_{v \in s_{0}}\left(T\left(\mathfrak{f}_{v}\right)\right) / \overline{T(\mathfrak{f})}$, а замыкание берется уже в $\prod_{v \in s_{0}} T\left(\mathfrak{f}_{v}\right)$. Нам достаточно показать, что $A\left(S, s_{0}\right)=0$. Пусть $x \in \prod_{v \in s_{0}} S\left(\mathfrak{f}_{v}\right) \quad$ и $y=\beta_{1}(x)$. Так как $\alpha_{1}-$ эпиморфизм, то существует $z \in H^{1}(\mathfrak{f}, A)$ такой, что $y=\alpha_{1}(z)$. Пусть $z=\gamma_{1}(t)$ при $t \in S(\mathfrak{f})$, тогда $y=\alpha_{1} \gamma_{1}(t)=$ $=\beta_{1} \alpha_{2}(t)=\beta_{1}(x)$. Следовательно, элемент $\quad r=\left[\alpha_{2}(t)\right]^{-1} x \in \prod_{v \in s_{0}} S\left(\mathfrak{f}_{v}\right)$ и, как видно, $\beta_{1}(r)=\left[\beta_{1}(x)\right]^{-1} \beta_{1}(x)=1$. Значит, $r=\beta_{2}(u)$ при некотором $u \in \prod_{v \in s_{0}} R\left(\mathfrak{f}_{v}\right)$ и $x=\alpha_{2}(t) \beta_{2}(u)$. По результату $M$. Кнезера [ $\left.{ }^{8}\right]$ $A(R)=0$, так как тор $R$ рационален. Это следует и из работы $\left[{ }^{2}\right]$. Поэтому элемент $u \in \prod_{v \in s_{0}} R\left(\mathfrak{f}_{v}\right)$ может быть аппроксимирован с любой степенью точности элементами $u_{n}$ из $R(\mathfrak{f})$. Тогда $x$ может быть аппроксимирован с любой степенью точности элементами $t \gamma_{2}\left(u_{n}\right) \in S(\mathfrak{f})$. Значит, $A\left(S, s_{0}\right)=0$.

В работе $\left[{ }^{2}\right]$ показано, что произвольный тор $T$ можно вложить в следующую точную последовательность:

$$
1 \rightarrow \operatorname{Pic} \bar{V} \hat{(T)} \rightarrow M \rightarrow T \rightarrow 1
$$

Свойства тора $M$ аналогичны свойствам тора $R$. Поэтому, если в тео- 
реме 2 вместо последовательности (3) исходить из последовательности (4), для произвольного тора получим следующий результат.

Теорем а 3. $A(T)=0$ тогда и только тогда, когда гомоморфизм $\varphi: H^{1}(\mathfrak{f}, \mathrm{Pic} \bar{V}(T)) \rightarrow \prod_{v \in s_{0}} H^{1}\left(\mathfrak{f}_{v}, \mathrm{Pic} \widehat{\bar{V}(T)}\right)$ является эпиморфизмом.

3 а меч ани е 2. В. Е. Воскресенский в работе $\left[{ }^{2}\right]$ доказал теорему о том, что $A(T)=0$, если $H^{1}\left(\mathfrak{f}_{v}, \operatorname{Pic} \bar{V}(T)\right)=0$ для всех $v \in s_{0}$. Но по локальной двойственности Тэйта $H^{1}\left(\mathfrak{f}_{v}, \operatorname{Pic} \bar{V}(T)\right)=H^{1}\left(\mathfrak{f}_{v}, \operatorname{Pic} \bar{V}(T)\right)$. Теорема 3 , следовательно, является обобщением этого результата.

В заключение отметим, что для тора $S$ из задачи погружения эквивалентны следующие утверждения:

1. $A(S)=0$.

2. Гомоморфизм $\alpha_{1}$ является эпиморфизмом.

3. Гомоморфизм ф является эпиморфизмом.

3 а меч ание 3 . Множество $s_{0}$ в теоремах 2 и 3 может быть заменено на любое конечное множество нормирований поля $\mathfrak{f}$, содержащее $s_{0}$.

\section{ЛИТЕРАТУРА}

1. O n o T., Ann. Math., 78, 47 (1963).

2. В оск ресен нки й В. Е., Изв. АН СССР, Сер. матем., 34, 3 (1970),

3. В оск ресенский В. Е., Изв. АН СССР, Сер. матем., 38, 3 (1974).

4. В ейл ь А., В сб.: Математика, 8, № 4, 3 (1964).

5. Б аш м а о в М. И.. Матем. заметки, 4, вып. 2, 1968 , с. 137.

6. Ф а д де е в Д. К., ДАН СССР. ХCIV, № 6, 1013 (1954).

7. Ш а фа р е в и и И. Р., ДАН СССР, XCV, № 3, 459 (1954).

8. Kneser M., Schwache Approximation in algebraischen Gruppen. Colloq. sur la théorie des groupes algèbre, Bruxelles, 1962.

Ленинградский государственный университет им. А. А. Жданова

Поступила в редакцию 28/V 1976

\section{A. KRJUTSKOV}

\section{UHE ALGEBRALISTE RONGASPINDIADE KLASSI ARITMEETILISED INVARIANDID}

\section{A. KRYUCHKOV}

\section{ARITHMETICAL INVARIANTS OF ONE CLASS OF ALGEBRAIC TORI}

Some recent results on algebraic tori are applied to tori arising in number field embedding problem with Abelian kernel. The kernel on weak approximation of such tori is investigated, and one common criterium is found. Besides that, some new cases are found when the Faddeyeff-Hasse necessary condition is sufficient for solving this problem. 